\title{
The application of methylene blue coloration technique in axillary lymph node dissection of breast cancer
}

\author{
Jieya Zou, Xiaoqi Wang, Zhuangqing Yang, Xiaojuan Yang, Chang'an Wang, Lifei Sun, Wenhuan Wang, \\ Yue Wang, Jianyun Nie
}

Third Department of Breast Surgery, The Third Affiliated Hospital of Kunming Medical University, Yunnan Cancer Hospital, Kunming 650118, China

Contributions: (I) Conception and design: All authors; (II) Administrative support: Z Yang, X Yang, C Wang; (III) Provision of study materials: J Zou, X Wang; (IV) Collection and assembly of data: All authors; (V) Data analysis and interpretation: All authors; (VI) Manuscript writing: All authors; (VII) Final approval of manuscript: All authors.

Correspondence to: Jianyun Nie. Third Department of Breast Surgery, The Third Affiliated Hospital of Kunming Medical University, Yunnan Cancer Hospital, Kunming 650118, China. Email: njyvip@sina.com.

Background: To analyze the associated factors that affect the coloration of methylene blue (MB) in axillary lymph node dissection (ALND) of patients with breast cancer and to explore the tracer effect of MB in high axillary lymph node metastasis, to guide surgical treatment.

Methods: We recruited 170 patients who underwent ALND, all of them were injected MB before operation. We analyzed the relationships between the clinical factors of age, body mass index (BMI), molecular typing, TNM staging, neoadjuvant chemotherapy, injection time and position and the MB coloration by univariate and multivariate analyses. A total of 84 cases were selected for observation of the application value of $\mathrm{MB}$ during intervention involving the lymph nodes upper axillary vein.

Results: Of 170 cases, 138 cases $(81.17 \%)$ were colored. Univariate analysis showed that significant differences were observed between differing BMI's $\left(\chi^{2}=24.074, \mathrm{P}<0.0001\right)$ and injection times $\left(\chi^{2}=41.207\right.$, $\mathrm{P}<0.0001)$. Multivariate analysis showed that injection time $(\mathrm{P}=0.016)$ was the clinical factor associated with $\mathrm{MB}$ coloration. More than 60 minutes before surgery and MB injection, the possibility of methylene blue colored was 0.088 times higher than 10 minutes $(\mathrm{P}=0.010,95 \% \mathrm{CI}, 0.014,0.554)$. MB was used to track the lymph node upper axillary vein with a sensitivity of $12.5 \%$, a specificity of $100 \%$, a false negative rate of $87.50 \%$, and a false positive rate of 0 ; the kappa coefficient was $0.051\left(\chi^{2}=0.679, \mathrm{P}=0.404\right)$.

Conclusions: Among the clinical factors, MB coloration was worse more than 60 minutes before ALND. Using the technology of MB color, lymph nodes can be clearly identified. It has great guiding value for the doctors who learn the operation initially. However, it is still prudent to use MB for high lymph node dissection.

Keywords: Methylene blue (MB); breast cancer; axillary lymph node dissection (ALND); the lymph node upper axillary vein

Submitted Jun 11, 2019. Accepted for publication Oct 10, 2019.

doi: $10.21037 /$ tcr.2019.10.42

View this article at: http://dx.doi.org/10.21037/tcr.2019.10.42

\section{Introduction}

In recent years, because of higher incidence, more attention has been given to breast cancer. It has been reported that breast cancer is the most common cancer among women in many Chinese cities (1). Surgery always plays an important role in the treatment of breast cancer. No matter how the method changes or the extent of the breast cancer surgery, ALND remains the standard surgical treatment for nodepositive disease. It is performed to confirm the axillary 


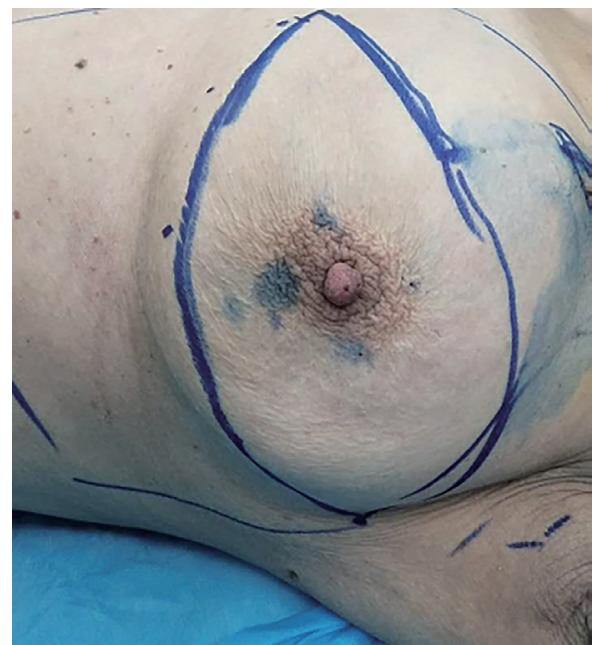

Figure 1 To inject methylene blue in areola area.

lymph node status, which is important as a prognostic factor for breast cancer (2-4).

For classification of lymph node stations within the axilla, the description of three axillary levels, defined by the pectoralis minor muscle, prevails. In general, axillary lymph node metastasis obeys the laws of levels I to II to III; however, there are also some that do not follow this rule, called Skip Metastasis, of which the incidence is $1.5-19.2 \%$ (5). For the axillary lymph nodes of high areas (called high lymph nodes in our research), patients treated with clearing this area are at the greatest risk for developing impairments of the arm and shoulder; upper limb lymphedema, pain, and sensitivity disorders were reported most frequently (6) Although controversial, it is considered that the lymph nodes with higher positions also belong to the lymphatic drainage area of axilla and that there is a risk of skip metastasis, which makes its removal necessary (7). Therefore, it is of great significance to identify and remove the lymph nodes with higher positions.

Using the appropriate lymph tracer can improve the detection rate of sentinel lymph nodes as well as biopsy rates (8). Methylene blue (MB) can be used widely in sentinel node biopsies (SLNB), as it is characterized by cost effectiveness, avirulence, long residence times in lymph nodes; reports have shown that there is no significance in using MB alone in SLNB versus using a combined isotope tracer (9). Initial investigators used blue dye to identify the ARM nodes or lymphatics. The concept of ARM involves mapping the lymphatic drainage from the upper extremity to determine the anatomical lymphatic variation and thus provides a roadmap to preserving these nodes during
ALND, thereby minimizing arm lymphedema $(10,11)$.

Over the past decade, MB has been used widely in clinics, and it has a good effect on the SLNB tracer $(9,12)$. However, researchers have been less enthusiastic about using MB in high lymph nodes in ALND. There is no consensus among researchers on clinical factors that affect the coloration of $\mathrm{MB}$, and this missing conclusion has important guiding significance to clinical practice $(13,14)$.

The present clinical trial was designed to analyze injection time, position and age, BMI, molecular typing, TNM staging, and neoadjuvant chemotherapies that affect the coloration of MB. The study was also designed to analyze the application of $\mathrm{MB}$.

\section{Methods}

\section{Patients}

We studied 170 patients diagnosed with breast cancer from December 2016 to December 2017 in the Department of Second Breast Surgery of Yunnan Cancer Hospital.

A total of 136 cases were diagnosed as invasive ductal carcinoma, accounting for $80 \%$ of the cases. There were 8 cases of invasive lobular carcinoma and 15 cases of mucinous adenocarcinoma, accounting for $4.70 \%$ and $8.82 \%$, respectively. The remaining 11 cases of breast cancer accounted for $6.47 \%$ of the enrolled patients. Basic demographic information, clinical diagnosis, treatment information and laboratory test information were collected by consulting medical records. Information collection strictly followed the principle of informed consent and the principle of patient information confidentiality. The ethics review of this study was completed by the medical ethics committee of Kunming Medical University (no: 2016-030-KJ-QT).

\section{Injection of MB injection position}

The positions of MB injection are the areola area (Figure 1) and the outer-upper quadrant of the breast (Figure 2). If patients were afflicted with tumors located in the outerupper quadrant, with "local excision" or "biopsy", injection of MB could be done outside of the selected area, away from the tumor, using $75 \%$ alcohol to disinfect the areola area in preparation for 3, 6, 9, and 12 o'clock injection sites of $0.5 \mathrm{~mL} \mathrm{MB}$. Injection of $2 \mathrm{~mL} \mathrm{MB}$ into the outerupper quadrant of breast was done to make sure the dot of injection is within the scope of the mastectomy. 


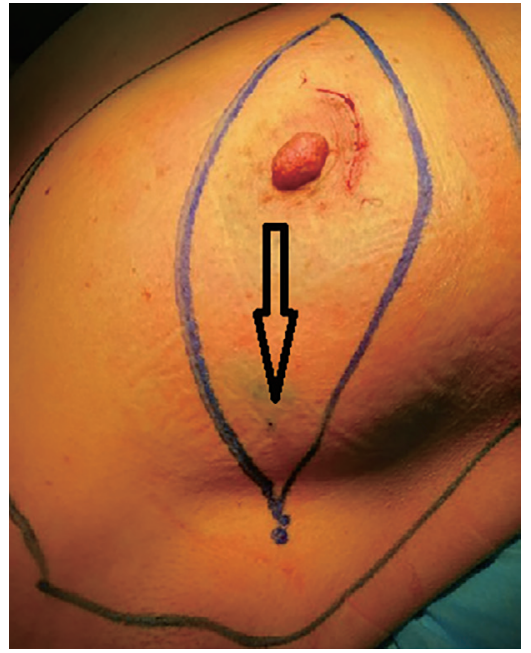

Figure 2 To inject methylene blue in the upper quadrant of breast.

\section{Injection time}

A total of 170 patients were divided into seven groups randomly; the groups were defined by minutes between the MB injection time and the ALND: 10, 20, 30, 40, 60, 120, and 240 minutes before ALND. After injection, the injection site was gently massaged with a medical cotton ball to facilitate the introduction of MB into the lymphatic vessels for drainage. Coloration of the axillary lymph nodes and lymphatics was observed.

\section{Operation methods}

After the anesthesia had taken effect, the patient was in a supine position, and her limbs in brackets, cleaning of the levels I and II lymph nodes was performed and then, cleaning of the lymph nodes of the upper axillary vein was performed. Using an ultrasonic knife, the upper axillary vein was fully exposed; having upward separation, we can see a "palisade" area, called the "upper axillary vein lymph node", noticeably clearing and packing the tissue. It was necessary to pay more attention to preserve blood vessels and nerves. After surgery, relevant pathological data were collected.

\section{Statistical analysis}

\section{Analysis of clinical factors}

To analyze the clinical factors that affected the coloration of $M B$, first, a single factor analysis was carried out using a $\chi^{2}$ test. In addition to this single factor analysis of $\mathrm{P}<0.1$, the reports of $\mathrm{MB}$ color influence factors (13-16) were fed into a Logistic regression model for multivariate analysis; we set $\mathrm{MB}$ color as the dependent variable $(1=$ not color, $2=$ color $)$.

To analyze the application of MB in the lymph node upper axillary vein

We evaluated the tracer effect of $M B$, compared with the biopsy results. We calculated sensitivity (the positive percentage of cases $\mathrm{MB}$ colored in axillary lymph node metastasis), specificity (the percentage of cases $\mathrm{MB}$ uncolored in axillary lymph node without metastasis), false negative rate (1-sensitivity) and false positive rate (1-specificity) to evaluate its accuracy. We calculated the kappa coefficient to evaluate its reliability: $K=1$ for both completely consistent, $0.4<\mathrm{K}<0.75$ for both moderately consistent, $\mathrm{K} \geq 0.75$ for both excellently consistent, $\mathrm{K} \leq 0.4$ for both poorly consistent, when $\mathrm{K}<0.4$; we did the $\chi^{2}$ test. SPSS 19.0 software was used for statistical analysis, and the alpha was set to $\alpha=0.05$.

\section{Results}

\section{Clinical factors that affect the coloration of MB patients}

A total of 170 patients were enrolled in the study; their mean age was $50.58 \pm 10.35$ years, the minimum age was 27 , and the maximum age was 85 . The mean body mass index (BMI) was $23.55 \pm 3.32$. In the clinical stage, there were 16 patients in stage I $(9.41 \%), 118$ patients in stage II (69.41\%), 24 patients in stage III $(14.12 \%)$ and 6 patients in stage IV (3.53\%); 6 patients' (3.53\%) stages were not confirmed. Concerning the molecular typing of breast cancer, there were 40 patients of Luminal A (23.53\%), 79 patients of Luminal B (46.47\%), 24 patients of ERBB2+ (14.12\%), and 27 patients of Basal-like (15.88\%). A total of 138 patients with axillary lymph nodes and lymphatic vessels showed blue (colored group, $81.18 \%$ ), 32 cases did not show blue (uncolored group, 18.82\%) (Figures 3-5).

\section{The results of single factor analysis}

The results of single factor analysis showed that BMI $\left(\chi^{2}=24.074, P<0.0001\right)$ and injection time $\left(\chi^{2}=41.207\right.$, $\mathrm{P}<0.0001)$ were the clinical factors that affected the coloration of MB. Age, BMI, molecular typing, TNM staging, neoadjuvant chemotherapy and the injection position had no significant influence on the coloration of MB $(\mathrm{P}>0.05)$. BMI $\left(\chi^{2}=24.074, \mathrm{P}<0.0001\right)$ (Table 1). 


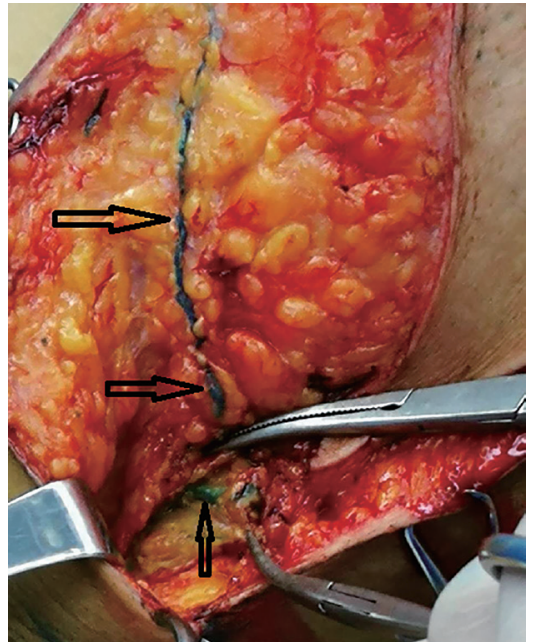

Figure 3 Lymphatic vessels and lymph nodes that were shown to be colored by methylene blue in ALND. ALND, axillary lymph node dissection.

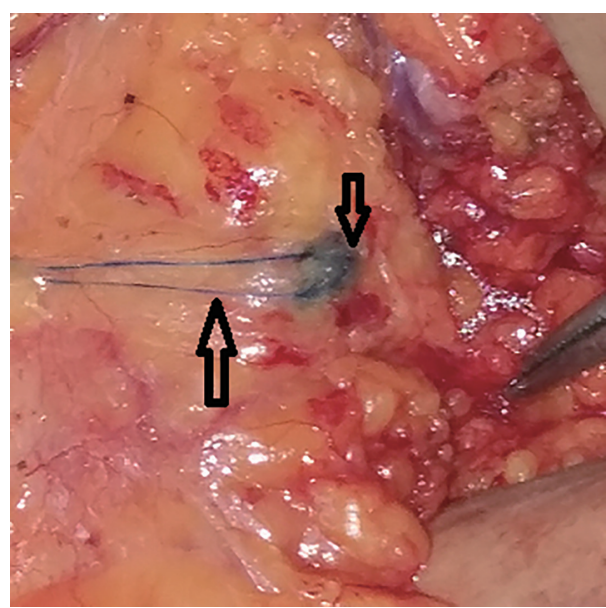

Figure 4 Lymphatic vessels and lymph nodes that were shown to be colored by methylene blue in ALND. ALND, axillary lymph node dissection.

\section{The results of multivariate analysis}

The results of multivariate analysis showed that injection time $(\mathrm{P}=0.016)$ was the clinical factor that affected the coloration of MB. Injection of MB more than 60 minutes before surgery resulted in 0.088 times higher than 10 minutes before surgery ( $\mathrm{P}=0.010,95 \% \mathrm{CI}, 0.014,0.554)$ (Table 2).

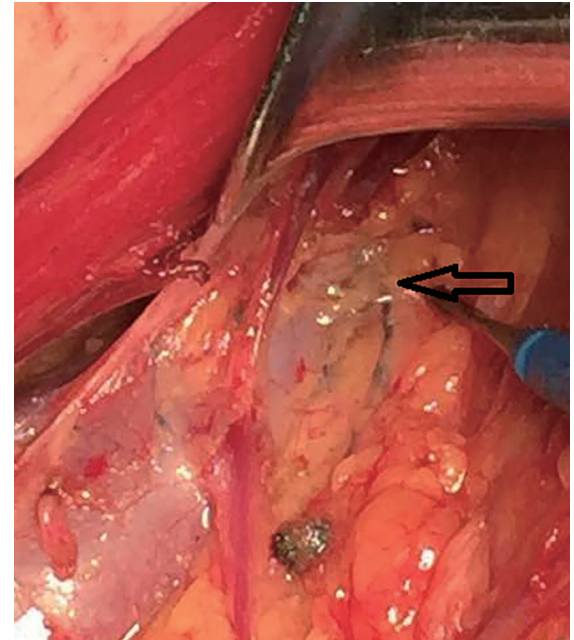

Figure 5 Methylene blue is seen crossing the axillary vein along the lymphatic duct.

\section{The application of MB in the lymph nodes of the upper axillary vein}

In this study, 84 cases were selected to for observation of the lymph nodes of the upper axillary vein. In some cases, $\mathrm{MB}$ is seen crossing the axillary vein along the lymphatic duct (Figure 5).

The results showed that 40 patients had no axillary lymph node metastasis, and 44 patients had axillary lymph node metastasis. Among the 84 patients, 69 cases of MB showed blue coloration. A total of 62 cases had no lymph node metastasis in the upper axillary vein, while 7 cases had lymph node metastasis upper axillary vein (Table 3).

MB was used to track the lymph nodes upper axillary vein with a sensitivity of $12.5 \%$, a specificity of $100 \%$, a false negative rate of $87.50 \%$, a false positive rate of 0 , and a kappa coefficient of $0.051\left(\chi^{2}=0.679, \mathrm{P}=0.404\right)$.

\section{Discussion}

An excellent stain, such as MB, to be used for analysis of the lymphatic system and lymph nodes should be characterized by cost effectiveness, avirulence, long residence times in the tissues and color clarity, among others. A large number of experiments have confirmed the accuracy and reliability of $\mathrm{MB}$, and it is widely used in clinics.

This study enrolled 170 cases, and 138 cases were 
Table 1 The results of single factor analysis of clinical factors that affect the coloration of methylene blue

\begin{tabular}{|c|c|c|c|c|c|}
\hline Clinical factors & Colored group, $\mathrm{N}=138(\%)$ & Uncolored group, $\mathrm{N}=32$ (\%) & Total, $\mathrm{N}=170(\%)$ & $\chi^{2}$ & $\mathrm{P}$ \\
\hline$\leq 40$ & $26(18.84)$ & $2(6.25)$ & $28(16.47)$ & & \\
\hline $41-50$ & 46 (33.33) & $14(43.75)$ & $60(35.29)$ & & \\
\hline $51-60$ & $44(31.88)$ & $13(40.63)$ & $57(33.53)$ & & \\
\hline BMI & & & & 24.074 & $<0.0001$ \\
\hline$<18.5$ & $10(7.25)$ & $0(0.0)$ & $10(5.88)$ & & \\
\hline $18.5-24.9$ & $91(65.94)$ & $9(28.13)$ & $100(58.82)$ & & \\
\hline 25.0-29.9 & $35(25.36)$ & 19 (59.38) & $54(31.76)$ & & \\
\hline Luminal A & $32(23.19)$ & $8(25.0)$ & $40(23.53)$ & & \\
\hline Luminal B & $61(44.20)$ & $18(56.25)$ & $79(46.47)$ & & \\
\hline ERBB2+ & $22(15.94)$ & $2(6.25)$ & $24(14.12)$ & & \\
\hline Basal-like & $23(16.67)$ & $4(12.50)$ & $27(15.88)$ & & \\
\hline TNM stage & & & & 1.875 & 0.759 \\
\hline Stage I & $12(8.70)$ & $4(12.50)$ & $16(9.41)$ & & \\
\hline Stage II & $96(69.57)$ & $22(68.75)$ & $118(69.41)$ & & \\
\hline Stage III & $21(15.22)$ & $3(9.38)$ & $24(14.18)$ & & \\
\hline Injection time (min) & & & & 41.207 & $<0.0001$ \\
\hline 10 & $28(20.29)$ & $2(6.25)$ & $30(17.65)$ & & \\
\hline 20 & $30(21.74)$ & $0(0.00)$ & $30(17.65)$ & & \\
\hline 30 & $28(20.29)$ & $2(6.25)$ & $30(17.65)$ & & \\
\hline 40 & $26(18.84)$ & $4(12.50)$ & $30(17.65)$ & & \\
\hline$\geq 60$ & $26(18.84)$ & $24(75.00)$ & $50(29.41)$ & & \\
\hline Injection position & & & & 1.079 & 0.299 \\
\hline Areola area & $95(68.84)$ & $25(78.13)$ & $120(70.59)$ & & \\
\hline The outer upper quadrant of breast & $43(31.16)$ & $7(21.88)$ & $50(29.41)$ & & \\
\hline
\end{tabular}


Table 2 The results of multivariate analysis of clinical factors that affect the coloration of methylene blue

\begin{tabular}{|c|c|c|c|c|c|c|}
\hline Clinical factors & Partial regression coefficients & Standard error & Wald value & $\mathrm{P}$ & OR & $95 \% \mathrm{Cl}$ \\
\hline \multicolumn{7}{|l|}{$\leq 40^{\star}$} \\
\hline $41-50$ & -2.046 & 1.043 & 3.849 & 0.05 & 0.129 & $(0.017,0.998)$ \\
\hline $51-60$ & -1.291 & 1.033 & 1.561 & 0.212 & 0.275 & $(0.036,2.084)$ \\
\hline \multicolumn{7}{|l|}{ BMI } \\
\hline \multicolumn{7}{|l|}{$<18.5^{\star}$} \\
\hline $18.5-24.9$ & -19.296 & $10,934.018$ & 0.0001 & 0.999 & - & - \\
\hline $25.0-29.9$ & -20.747 & $10,934.018$ & 0.0001 & 0.999 & - & - \\
\hline \multicolumn{7}{|l|}{ Molecular subtype } \\
\hline \multicolumn{7}{|l|}{ Luminal $A^{*}$} \\
\hline Luminal B & -0.515 & 0.667 & 0.58 & 0.446 & 0.597 & $(0.159,2.250)$ \\
\hline ERBB2+ & 1.189 & 1.132 & 1.103 & 0.294 & 3.283 & $(0.357,30.178)$ \\
\hline Basal-like & 0.294 & 0.956 & 0.095 & 0.758 & 1.342 & $(0.206,8.747)$ \\
\hline \multicolumn{7}{|l|}{ TNM stage } \\
\hline \multicolumn{7}{|l|}{ Stage $I^{*}$} \\
\hline Stage II & -0.084 & 0.896 & 0.009 & 0.925 & 0.92 & $(0.159,5.321)$ \\
\hline Stage III & 0.282 & 1.126 & 0.063 & 0.802 & 1.326 & $(0.146,12.052)$ \\
\hline 20 & 32.471 & $8,048.385$ & 0.0001 & 0.997 & - & - \\
\hline 30 & 0.425 & 1.205 & 0.125 & 0.724 & 1.53 & $(0.144,16.225)$ \\
\hline 40 & -0.864 & 1.046 & 0.681 & 0.409 & 0.422 & $(0.054,3.276)$ \\
\hline$\geq 60$ & -2.433 & 0.94 & 6.699 & 0.010 & 0.088 & $(0.014,0.554)$ \\
\hline Injection position & 1.315 & 0.794 & 2.743 & 0.098 & 3.726 & $(0.786,17.666)$ \\
\hline
\end{tabular}

${ }^{*}$, comparable group; ${ }^{*}, \mathrm{P}<0.05$.

colored, accounting for $81.17 \%$. Single factor analysis showed that BMI $\left(\chi^{2}=24.074, \mathrm{P}<0.0001\right)$ and injection time $\left(\chi^{2}=41.207, \mathrm{P}<0.0001\right)$ are clinical factors that affect the coloration of MB. Age, BMI, molecular typing, TNM staging, neoadjuvant chemotherapy and injection position had no significant influences on the coloration of $\mathrm{MB}$
( $P>0.05)$. The results of the multivariate analysis showed that injection time $(\mathrm{P}=0.016)$ is a clinical factor that affects the coloration of $\mathrm{MB}$. Injection of $\mathrm{MB}$ more than 60 minutes before surgery resulted in 0.088 times higher the possibility of $\mathrm{MB}$ colored than 10 minutes before surgery $(\mathrm{P}=0.010,95 \% \mathrm{CI}, 0.014,0.554)$. We injected $\mathrm{MB}$ 
Table 3 The diagnosis results that axillary lymph node metastasis upper axillary vein by methylene blue tracer

\begin{tabular}{lcccc}
\hline \multirow{2}{*}{ Methylene blue tracer } & \multicolumn{3}{c}{ Biopsy } & Total \\
\cline { 2 - 4 } & Metastasis & Not metastasis & 7 \\
Positive & 7 & 0 & 62 \\
Negative & 49 & 13 & 69 \\
Total & 56 & 13 & 69 \\
\hline
\end{tabular}

in the areola area or the outer-upper quadrant of the breast; injection site was not shown to be a factor that affects the coloration of MB. The results are similar to some published studies, which investigated the use of MB in SLNB (15-20). MB can be a significant guide when identifying clear lymphatic vessels and lymph nodes in ALND.

Several researchers used blue dye approximately five minutes before SLNB $(21,22)$. Sakurai et al. (23) performed surgery immediately after the injection of blue dye in ARM. In this study, the fastest time of separating skin and subcutaneous tissue in ALND was 10 minutes. The injection time of 10, 20 and 30 minutes can guarantee a $\mathrm{MB}$ color rate of above $90 \%$. As time goes on, the color effect decreases. Injection of $\mathrm{MB}$ more than 60 minutes before surgery resulted in 0.088 times higher the possibility of $\mathrm{MB}$ colored than 10 minutes before surgery. Therefore, the injection time should be less than 60 minutes when using MB to guide ALND.

In a meta-analysis published by Pesek et al. (24), there were seven categories based on location of injection, reported by 302 articles. Four categories consisted of a single location site, while three categories represented a combination of locations. There were no statistically significant variations between these seven location categories $(\mathrm{P}=0.95)$. Intra-tumor injections had the lowest numerical FNR at $2.5 \%$. Similarly, Ahmed et al. (25) also considered that there was no significant difference between superficial and deep injections of radioactive tracer for axillary SLN identification on lymphoscintigraphy. In our research, there was no statistical significance between areola and outer-upper quadrant for the injection of $\mathrm{MB}$ in ALND. If the clinical lesions were located in the outer area, a biopsy may be the cause of local tissue edema or lymphatic drainage structure change, which could affect the path of $\mathrm{MB}$ tracer. It is suggested that increasing injection quantity in the outer area or changing the injection site can improve the detection rate of lymph nodes. Nevertheless, it is a problem that the injection position diffusion zone with the axillary nodes is often overlapped with tumors located in the upper outer quadrant and axillary tail of the breast, or patients have undergone biopsies in the upper-outer quadrant of the breast. Thus, injecting $M B$ in the subareolar area is a better choice (26).

In this study, 84 cases were selected for observation of the lymph nodes in the upper axillary vein. A total of 69 cases of MB showed blue. A total of 62 cases had no lymph node metastasis in the upper axillary vein, and 7 cases had lymph node metastasis in the upper axillary vein. $\mathrm{MB}$ was used to track the lymph nodes in the upper axillary vein and showed a sensitivity of $12.5 \%$, a specificity of $100 \%$, a false negative rate of $87.50 \%$, a false positive rate of 0 , and a kappa coefficient of $0.051\left(\chi^{2}=0.679, \mathrm{P}=0.404\right)$. The results showed that methylene blue trace has a high false negative rate, low sensitivity, and poor consistency. We suggest that $\mathrm{MB}$ can serve as an excellent guide for identifying and clearing lymphatic vessels and lymph nodes in ALND; however, it is still prudent to use MB for high lymph node dissection.

It is reported that the detection rate of SLN was $85-95 \%$, the accuracy rate was $71-98 \%$, and the false negative rate (FNR) was $0-29 \%$ when $\mathrm{MB}$ was used in SLNB $(9,27)$. In ALND, MB is used to perform axillary reverse mapping (ARM); different identification rates (DR) are reported for ARM nodes ranging from $65 \%$ to $82 \%$ by different investigators $(11,28)$. In the meta-analysis published by Kim et al. (29), the mean identification rate was $83.1 \%$ with blue dye alone and $96.4 \%$ with dye and a tracer. There was no difference between tracer-only and dye-only $(\mathrm{P}=0.370)$, or tracer-only and dye-and-tracer $(\mathrm{P}=0.178)$. The lymph node upper axillary vein almost covers the high lymph nodes of axilla, which are rich in blood supply and of great significance. It has been confirmed $(30,31)$ that blood vessels and tumor angiogenesis are generally associated with tumor growth and poor clinical outcome in cancer patients. Angiogenesis, the formation of neo-vessels, is one of the most important hallmarks of cancer. Therefore, the lymph 
nodes' upper axillary veins were taken as target areas in this study. Axillary lymph nodes of levels I, II, III are artificial groups; three levels are in the same station on transfer behavior, and three levels may be the first station for breast cancer metastatic lymph nodes. It is controversial to clean level III axillary lymph nodes. The lymph node upper axillary vein in our study, as well as axillary level III lymph nodes on the anatomy, is higher in the area of axilla. Some scholars believe that the higher area upper axillary vein belongs to the brachial plexus lymphatic drainage area. Lymphedema, dysfunction, bleeding risk and even destruction of the axillary vein scabbard are connected with cleaning the lymph node upper axillary vein. Other scholars argue that failing to clean the lymph node upper axillary vein may downstage inadequate treatment similar to level-III axillary lymph node $(7,32)$. We used MB to guide the cleaning of the lymph node upper axillary vein. Because of the high position, $\mathrm{MB}$ color was required to move along the lymphatic longer, and $\mathrm{MB}$ required a longer time to color, which may have affected the coloration. Therefore, we should pay more attention when cleaning the high lymph node with $M B$ tracer.

One of the main limitations of the current study was the use of MB. MB has some major disadvantages (33), such as allergic reactions, long time staining at the injection site and that proper use requires a certain training period. This method may be preferable for all clinicians if its accuracy is demonstrated to be comparable to that of the combination method. In addition, we analyze the clinical factors that affect the coloration of MB. BMI was found to be influential in the single-factor analysis, while no factors of influence were found in the multiple- factor analysis of our research. Proposed explanations for this are different measuring times and that we did not require patients to take off their shoes or remove clothing. These factors should be improved upon in subsequent studies.

Our study has shown that MB can be used in ALND and that it has great guiding value to novices of this surgery and primary hospitals. However, it is still prudent to use $\mathrm{MB}$ for high lymph node dissection. In breast cancer, MB has a great deal of advantages for SLNB. We believe that MB also has wide application prospects in ALND.

\section{Conclusions}

Among the clinical factors, $\mathrm{MB}$ coloration was worse more than 60 minutes before ALND. Using the technology of MB color, lymph nodes can be clearly identified. It has great guiding value for the doctors who learn the operation initially. However, it is still prudent to use MB for high lymph node dissection.

\section{Acknowledgments}

We thank Xiaowen Wang from Chinese Centers for Disease Control and Prevention for statistical analysis or interpretation. And the team named Science and Technology Innovation Team of Yunnan Province (2018HC002) supported us a lot. We thank all the individuals in the study for their participation.

Funding: This study is supported by a National Natural Science Foundation of China (No. 81760480).

\section{Footnote}

Conflicts of Interest: All authors have completed the ICMJE uniform disclosure form (available at http://dx.doi. org/10.21037/tcr.2019.10.42). The authors have no conflicts of interest to declare.

Ethical Statement: The authors are accountable for all aspects of the work in ensuring that questions related to the accuracy or integrity of any part of the work are appropriately investigated and resolved. The study was conducted in accordance with the Declaration of Helsinki (as revised in 2013). All patients were informed about the study objectives, and they were assured of confidentiality. They were asked to indicate their agreement and understanding with a signed informed consent form before the investigation. The study was approved by the ethics research committee of Kunming Medical University, China (Reference number: 2016-026-KJ-QT).

Open Access Statement: This is an Open Access article distributed in accordance with the Creative Commons Attribution-NonCommercial-NoDerivs 4.0 International License (CC BY-NC-ND 4.0), which permits the noncommercial replication and distribution of the article with the strict proviso that no changes or edits are made and the original work is properly cited (including links to both the formal publication through the relevant DOI and the license). See: https://creativecommons.org/licenses/by-nc-nd/4.0/.

\section{References}

1. Siegel RL, Miller KD, Jemal A. Cancer statistics, 2019. 
CA Cancer J Clin 2019;69:7-34.

2. Giuliano AE, Han SH. Local and regional control in breast cancer: role of sentinel node biopsy. Adv Surg 2011;45:101-6.

3. Dominici LS, Morrow M, Mittendorf E, et al. Trends and Controversies in Multi-Disciplinary Care of the Breast Cancer Patient. Curr Probl Surg 2016;53:559-95.

4. D'Angelo-Donovan DD, Dickson-Witmer D, Petrelli NJ. Sentinel lymph nodebiopsy in breast cancer: a history and current clinical recommendations. Surg Oncol 2012;21:196-200.

5. Sun J, Yina J, Nin L, et al. Clinicopathological characteristics of breast cancers with axillary skip metastases. J Invest Surg 2012;25:33-6.

6. Fleissig A, Fallowfield LJ, Langridge CI, et al. Postoperative arm morbidity and quality of life. Results of the ALMANAC randomised trial comparing sentinel node biopsy with standard axillary treatment in the management of patients with early breast cancer. Breast Cancer Res Treat 2006;95:279-93.

7. Dillon MF, Advani V, Masterson C, et al. The value of level III clearance in patients with axillary and sentinel node positive breast cancer. Ann Surg 2009;249:834-9.

8. Zakaria S, Hoskin TL, Degnim AC. Safety and technical success of methylene blue dye for lymphatic mapping in breast cancer. Am J Surg 2008;196:228-33.

9. Varghese P, Mostafa A, Abdel-Rahman AT, et al. Methylene bluedye versus combined dye-radioactive tracer technique for sentinellymph node localisation in early breast cancer. EJSO 2007;33:147-52.

10. Tausch C, Baege A, Dietrich D, et al. Can axillary reverse mapping avoid lymph edema in node positive breast cancer patients. Eur J Surg Oncol 2013;39:880-6.

11. Noguchi M. Axillary reverse mapping for preventing lymph edema in the axillary lymph node dissection and/or sentinel lymph node biopsy.Breast Cancer 2010;17:155-7.

12. Özdemir A, Mayir B, Demirbakan K, et al. Efficacy of Methylene Blue in Sentinel Lymph Node Biopsy for Early Breast Cancer. J Breast Health 2014;10:88-91.

13. Boughey JC, Suman VJ, Mittendorf EA, et al. Factors affecting sentinel lymph node identification rate after neoadjuvant chemotherapy for breast cancer patients enrolled in ACOSOG Z1071 (Alliance). Ann Surg 2015;261:547-52.

14. Gschwantler-Kaulich D, Keil MR, Ruecklinger E, et al. Factors influencing the identification rate of the sentinel node in breast cancer. Eur J Cancer Care (Engl)
2011;20:627-31.

15. Chagpar AB, Martin RC, Scoggins CR, et al. Factors predicting failure to identify a sentinel lymph node in breast cancer. Surgery 2005;138:56-63.

16. Kang T, Yi M, Hunt KK, et al. Does Blue Dye Contribute to Success of Sentinel Node Mapping for Breast Cancer? Ann Surg Oncol 2010;17:280-5.

17. Eitan R, Sabah G, Krissi H, et al. Robotic blue-dye sentinel lymph node detection for endometrial cancerFactors predicting successful mapping. Eur J Surg Oncol 2015;41:1659-63.

18. Diego EJ, McAuliffe PF, Soran A, et al. Axillary staging after neoadjuvant chemotherapy for breast cancer: a pilot study combining sentinel lymph node biopsy with radioactive seed localization of pretreatment positive axillary lymph nodes. Ann Surg Oncol 2016;23:1549-53.

19. Hicks M, Macrae ER, Abdel-Rasoul M, et al. Neoadjuvant dual HER2-targeted therapy with lapatinib and trastuzumab improves pathologic complete response in patients with early stage HER2-positive breast cancer: a meta-analysis of randomized prospective clinical trials. Oncologist 2015;20:337-43.

20. Kuehn T, Bauerfeind I, Fehm T, et al. Sentinel-lymphnode biopsy in patients with breast cancer before and after neoadjuvant chemotherapy (SENTINA):a prospective, multicentre cohort study. Lancet Oncol 2013;14:609-18.

21. Takamaru T, Kutomi G, Satomi F, et al. Use of the dyeguided sentinel lymph node biopsy method alone for breast cancer metastasis to avoid unnecessary axillary lymph node dissection. Exp Ther Med 2014;7:456-60.

22. Guo W, Zhang L, Ji J, et al. Evaluation of the benefit of using blue dye in addition to indocyanine green fluorescence for sentinel lymph node biopsy in patients with breast cancer. World J Surg Oncol 2014;12:290.

23. Sakurai T, Endo M, Shimizu K, et al. Axillary reverse mapping using fluorescence imaging is useful for identifying the risk group of postoperative lymphedema in breast cancer patients undergoing sentinel node biopsies. J Surg Oncol 2014;109:612-5.

24. Pesek S, Ashikaga T, Krag LE, et al. The false-negative rate of sentinel node biopsy in patients with breast cancer: a meta-analysis. World J Surg 2012;36:2239-51.

25. Ahmed M, Purushotham AD, Horgan K, et al. Metaanalysis of superficial versus deep injection of radioactive tracer and blue dye for lymphatic mapping and detection of sentinel lymph nodes in breast cancer. Br J Surg 2015;102:169-81. 
26. James TA, Coffman AR, Chagpar AB, et al.

Troubleshooting Sentinel Lymph Node Biopsy in Breast Cancer Surgery. Ann Surg Oncol 2016;23:3459-66.

27. Naik AM, Fey J, Gemignani M, et al. The risk of axillary relapse after sentinel lymph node biosy for breast cancer is comparable with that of axillary lymph node dissection: a follow-up study of 4008 procedures. Ann Surg 2004;240:462-8; discussion 468-71.

28. Thompson M, Korourian S, Henry -Tillman R. Axillary reverse mapping (ARM): a new concept to identify and enhance lymphatic preservation. Ann Surg Oncol 2007;14:1890-95.

29. Kim T, Giuliano AE, Lyman GH. Lymphatic mapping

Cite this article as: Zou J, Wang X, Yang Z, Yang X, Wang C, Sun L, Wang W, Wang Y, Nie J. The application of methylene blue coloration technique in axillary lymph node dissection of breast cancer. Transl Cancer Res 2019;8(8):2781-2790. doi: $10.21037 /$ tcr.2019.10.42 and sentinel lymph node biopsy in early-stage breast carcinoma. Cancer 2006;106:4-16.

30. Carmeliet P. Angiogenesis in life, disease and medicine. Nature 2005;438:932-6.

31. Carmeliet P, Jain RK. Angiogenesis in cancer and other diseases. Nature 2000;407:249-57.

32. Cserni G. Sentinel node biopsy and axillary clearance in early breast cancer-An algorithm with explanations and queries. Magy Seb 2016;69:4-13.

33. Bézu C, Coutant C, Salengro A, et al. Anaphylactic response to blue dye during sentinel lymph node biopsy. Surg Oncol 2011;20:e55-9. 